\title{
RECOMENDACIONES EN NUTRICIÓN, DIETA Y SALUD BUCAL PARA LOS NIÑOS
}

ALVEAR-CÓRDOVA MARÍA *

Recibido: 10/12/2015

Aceptado: 21/12/2015

\section{RESUMEN}

El objetivo del presente artículo de opinión es presentar las recomendaciones de los expertos respecto a la nutrición y dieta adecuada para la salud bucal de los niños. Se tocan temas como la dieta sana para los dientes, la pirámide alimenticia, las recomendaciones de la Académica Americana de Odontopediatria respecto al asesoramiento y orientación a los padres respecto a cuidados dietéticos y de higiene oral para los lactantes y niños. Finalmente se sistematiza una serie de recomendaciones para los padres respecto al cuidado dental de los niños, basado en evidencia científica.

Palabras Clave: Dieta, alimentos, salud bucal.

\section{ABSTRACT}

The aim of this article is to present the opinion of experts on recommendations regarding nutrition and proper diet for oral health children's. This article take topics such as healthy diet, the food pyramid, the recommendations of the American Academy of Pediatric Dentistry respect to advice and guide parents regarding dietary and oral hygiene care for infants and children. Finally, it presents a series of recommendations for parents respect to dental care for children, based on scientific evidence

Keywords: Diet, food, oral health.

\section{I.- INTRODUCCIÓN}

La dieta y la nutrición desempeñan un papel equitativo y conjunto en la salud bucal infantil. Esta representa mucho más que dientes útiles y bonitos, constituye una parte integral de la salud general y sustenta el equilibrio esencial del bienestar humano.

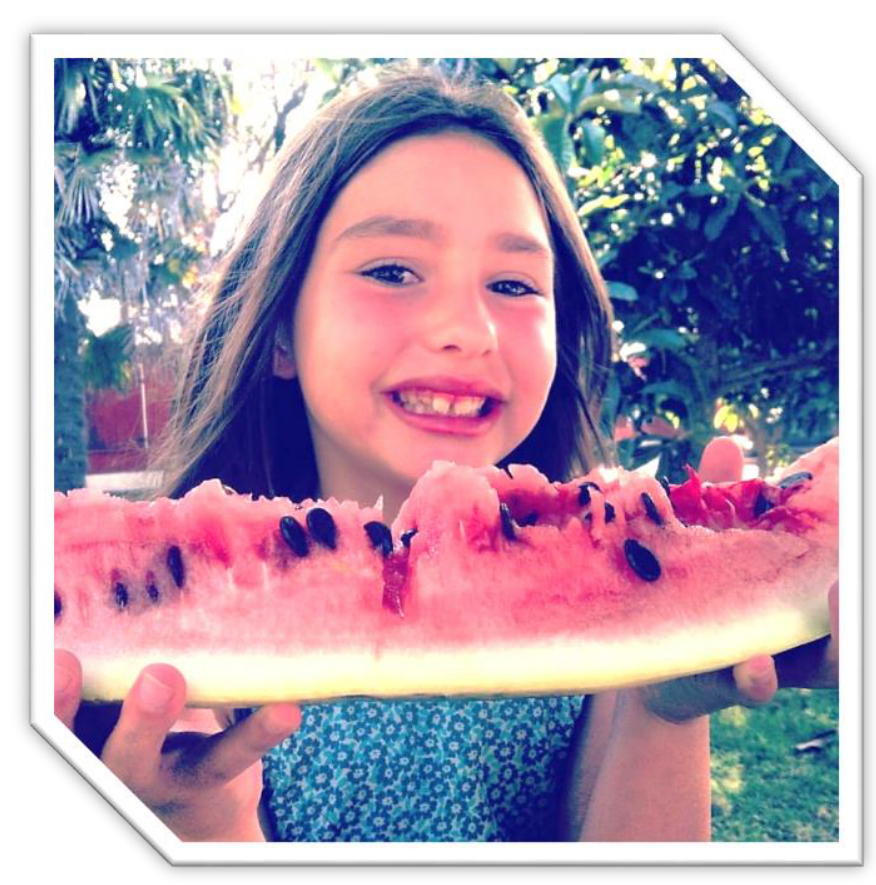

Figura 1. Elaboración propia

La buena nutrición del infante y una cavidad bucal con características saludables reflejan la existencia de una relación estrecha, por ello es posible sustentar que en cuanto a la salud bucal, el efecto más importante de la nutrición, es la acción local de la dieta y el papel que esta ejerce en el desarrollo de enfermedades como la caries dental, erosión dentaria, defectos del esmalte, enfermedades de la mucosa oral y enfermedad de encía.

Estudios de laboratorio y con intervención en humanos, muestran que el azúcar es el principal factor dietético asociado a la caries dental. ${ }^{1}$ Pese a las sofisticadas técnicas preventivas de hoy en día y el rol fundamental de los fluoruros, la caries no ha podido ser erradicada en su totalidad. Los patrones dietéticos inadecuados que incrementan la frecuencia de refrigerios y promocionan la ingesta de dulces y bebidas endulzadas han contribuido a un incremento en la incidencia de la enfermedad . ${ }^{2}$ Este patrón en general se ha mostrado más evidente en las familias de bajos ingresos económicos, lo cual podría estar relacionado con el costo superior de una dieta saludable, es así que estos hallazgos sugieren que el nivel socioeconómico es un posible obstáculo, para alcanzar hábitos alimenticios saludables. ${ }^{3}$

\footnotetext{
* Docente Especialista en Odontopediatria, Universidad Católica de Cuenca.Ecuador
} 
Las enfermedades dentales, han demostrado influencia directa en la calidad de vida de los niños, se han descrito impactos negativos de estas enfermedades en la capacidad de alimentación, dolor, ansiedad e incluso alteración en las relaciones sociales del niño. ${ }^{4}$ Este artículo tiene como objetivo proveer recomendaciones basadas en evidencia para los padres, de la relación existente entre la nutrición, dieta y la salud bucal de los infantes.

\section{II.-ESTADO DEL ARTE ${ }^{1,2,3}$}

\section{DIETA SANA PARA LOS DIENTES}

Una dieta sana es naturalmente equilibrada, y suministra todos los nutrientes que un niño necesita para crecer, incluye los siguientes grupos de alimentos: verduras, cereales, frutas, legumbres, carnes y leche. Una dieta excesivamente alta en hidratos de carbono, incrementa la posibilidad que un niño padezca caries dental. Figura 1.

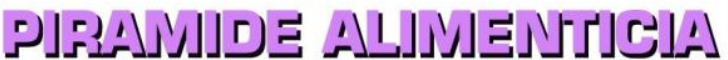

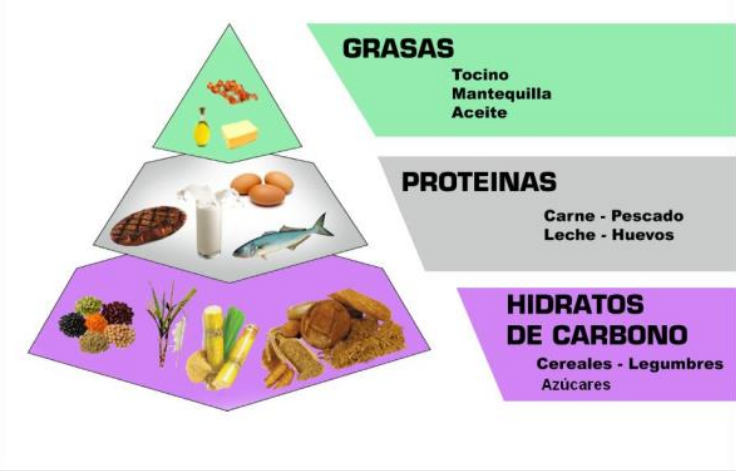

Figura 2. Elaboración Propia
El azúcar se puede encontrar en muchos alimentos procesados, incluso algunos que ni siquiera tienen sabor dulce. ${ }^{5}$ Por ejemplo, condimentos utilizados como aderezos de ensaladas, salsa de tomate, gelatinas, jaleas, helados, batidos, granizados, chocolates, papas fritas, pan, cereales para el desayuno y galletas saladas.

Estos alimentos reciben la denominación de cariogénicos, los cuales presentan características de fácil identificación para los padres, entre ellas: son pegajosos, de consistencia blanda, su consumo se lleva a cabo entre las comidas y no durante las mismas, adicionalmente su cariogenicidad se incrementa a mayor frecuencia de ingesta de los mismos. ${ }^{6}$ Figura 2.

Cuando los padres quieran comprobar cuan cariogénico es un alimento, deben mirar más allá de la azucarera y del plato de dulces. Una variedad de alimentos contienen uno o más tipos diferentes de azúcar, por ello es preciso enfatizar que todos los tipos de azúcar pueden fomentar la caries dental en mayor o menor grado. La prevención de la caries dental y otros problemas de salud oral, constituye un factor base en el manejo futuro del paciente dentro del área Odontopediátrica, puesto que se reducen notablemente cuadros dolorosos agudos y adicionalmente los costos asociados con el tratamiento.

La Academia Americana de Odontopediatría sugiere y enfatiza que el cuidado dental se inicia en el periodo prenatal con el asesoramiento y orientación precoz acerca de los cuidados dietéticos y de higiene oral para lactantes y niños. ${ }^{7}$

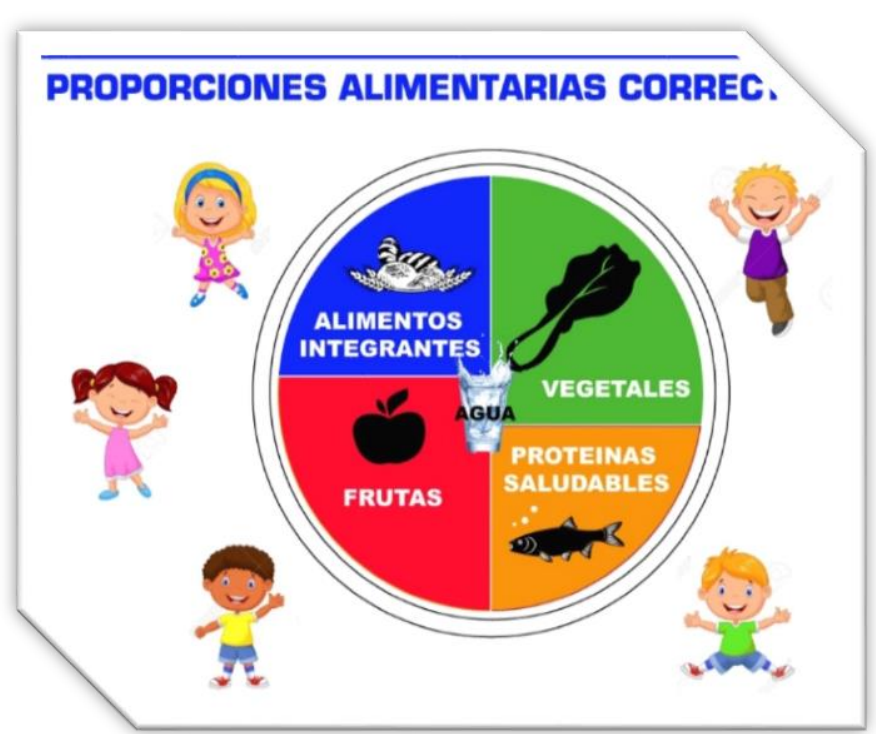

Figura 3. Elaboración Propia 
El enfoque planteado no sugiere que los niños deben renunciar a todos los alimentos con azúcar o almidón, ya que estos alimentos también forman parte de los nutrientes que se requieren para un crecimiento y desarrollo adecuado, por ello los padres son los encargados de seleccionarlos y servirlos a horarios racionalizados que generalmente no involucren periodos entre comidas los cuales quedan fuera del alcance de un cepillado cercano a la ingesta. Es decir los alimentos más azucarados son más seguros para los dientes si se incluyen dentro de una comida principal, más no como un aperitivo. ${ }^{8}$

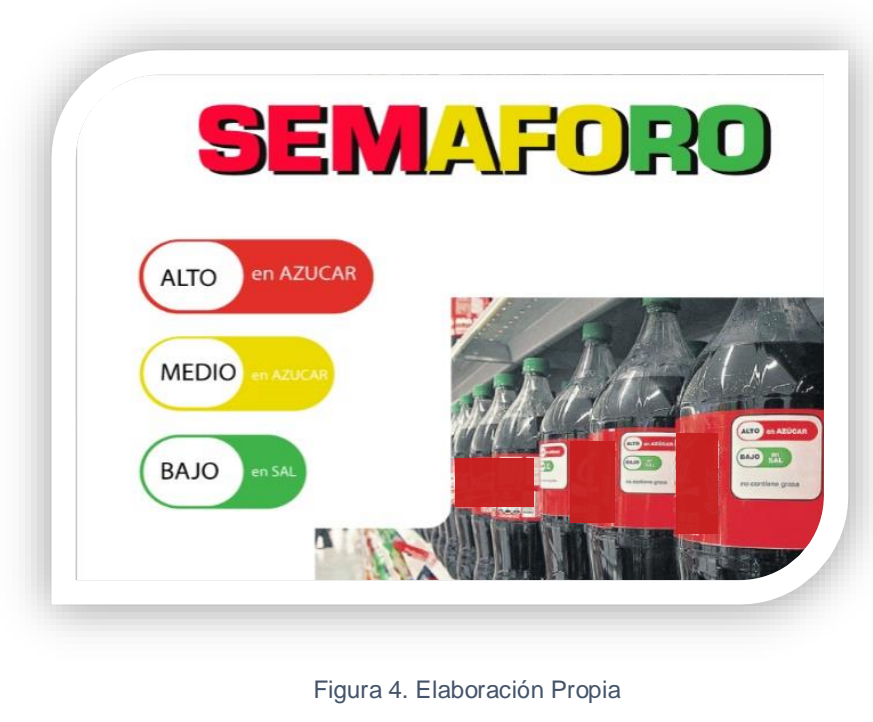

Con gran relevancia los padres deben conversar con un Pediatra y Odontopediatra para recibir la información acerca de la selección correcta de alimentos que protegen los dientes. Un Especialista debidamente capacitado en Odontología Pediátrica tiene la obligación de informar a los padres que existen alimentos como el pescado de mar, el agua y el té que contienen componentes protectores contra la caries, de los cuales el elemento preponderante es el flúor.

Otros componentes de notable efectividad aportan los alimentos que presentan calcio y fosfato en su composición entre los cuales destaca la leche y el queso, los cuales favorecen a procesos de refuerzo del esmalte. Por otro lado es preciso que sea de conocimiento de los padres que existen medicamentos de uso infantil cuyas fórmulas son ricas en azúcar (jarabes dulces), un previo análisis de la fórmula del medicamento podría prevenir enfermedades dentales y permitiría ofrecer otras alternativas de medicamentos libres de azúcar o cepillar los dientes inmediatamente después de tomar estos medicamentos. 9,10

\section{RECOMENDACIONES PARA LOS PADRES}

- Mantener en el niño una ingesta calórica adecuada, que apoye el crecimiento, desarrollo y peso normal del mismo. ${ }^{11}$

- La elección de una dieta rica en verduras, frutas, granos enteros, baja en grasas saturadas y colesterol, resulta una alternativa eficaz para mantener la salud oral y general del paciente pediátrico. Figura3.

- El uso de azúcares se debe controlar de manera moderada y racionalizada.

- La educación de los padres debe ser continua respecto a la asociación existente entre el consumo de hidratos de carbono y la presencia de caries dental. ${ }^{12}$

- Es esencial leer la información nutricional de las etiquetas de envasado de alimentos, lo cual puede modificar y estimular la selección de los mismos, orientando un estilo de vida saludable. ${ }^{13,14,15}$ Figura 4.

\section{III.- CONCLUSIONES}

Aunque cada niño es único, las ingestas y recomendaciones dietéticas de referencia, proporcionan directrices generales, que aseguran la proporción adecuada de nutrientes y limitan el riesgo de desarrollar caries. Todas las pautas dietéticas coinciden en recomendar el consumo de una variedad de alimentos de todos los grupos nutricionales. Los programas de educación para la salud y servicios de alimentación para promover programas nutricionales, son bases claves de las cuales deben beneficiarse los padres de infantes en pleno desarrollo. La investigación, la educación y la legislación apropiada deben trabajar de la mano en el desarrollo de dietas equilibradas que aseguren las características de salud integral en el paciente pediátrico. 


\section{REFERENCIAS BIBLIOGRÁFICAS}

1. Moynihan PJ. The role of diet and nutrition in the etiology and prevention of oral diseases. World Health Organization. 2005; 83:694-699.

2. Feldens CA, Rodrigues P.H, Rauber F. Food Expenditures, Cariogenic Dietary Practices and Childhood Dental Caries in Southern Brazil. Caries Res. 2013; 47:373-381.

3. Sankeshwari RM, Ankola AV. Association of socio-economic status and dietary habits with early childhood caries among 3- to 5-year-old children of Belgaum city. Eur Arch Paediatr Dent. 2013; 14:147-153.

4. Moyniha P, Petersen PE. Diet, nutrition and the prevention of dental diseases. Public Health Nutrition. $2004 ; 7(1 \mathrm{~A}): 201-226$.

5. American academy of Pediatric Dentistry. Ask your dentist about diet and snacking. 2011.

6. Hugar S, Hugar D, Sajjanshetty S. Diet Counselling For Pediatric Patient: A Review. Sch. J. App. Med. Sci. 2014; 2(4A):1199-1201.

7. Ogata B, Trahms C. Nutrition focus for oral children with special health care needs. Nutrition and Oral Health For children. 2003;18(6):2-9.

8. Collins MR, Laverty A, Roberts S. Eating behaviour and food choices in children with Down's syndrome, autistic spectrum disorder or cri du chat syndrome and comparison groups of siblings. JOLD. 2004; vol 8(4): 331-350.

9. Naidoo S. Oral health and nutrition for children under five years of age: a paediatric food-based dietary guideline. S Afr J Clin Nutr. 2013;26(3):S150-S155.

10. Marshall TA. Diet and nutrition in pediatric dentistry. Dent Clin N Am. 2003;47: 279-303.

11. American Academy Of Pediatric Dentistry. Policy on Dietary Recommendations for Infants, Children, and Adolescents. 2012;37(6):15-16.

12. Achahui AP, Albinagorta MJ, Arauzo CJ. Caries de Infancia temprana: diagnóstico e identificación de factores de riesgo. Odontol Pediatr. 2014;13(2):119-137.

13. Aarthi J, Muthu MS, Sujatha S. Cariogenic potential of milk and infant formulas: a systematic review. Eur Arch Paediatr Dent. 2013; 14:289-300.

14. Moursi AM, Fernandez JB. Nutrition and Oral Health Considerations in Children with Special Health Care Needs: Implications for Oral Health Care Providers. PEDIATRiC DENTISTRY. 2010;32(4):333342.

15. Milgrom $P$, Riedy $C A$, Weinstein $P$. Dental caries and its relationship to bacterial infection, hypoplasia, diet, and oral hygiene in 6- to 36-month-old children. Community Dent Oral Epidemio.I 2000; 28: 29530 . 